\title{
Determinants of the uptake of intermittent preventive treatment of malaria in pregnancy with sulphadoxine pyrimethamine in Sabatia Sub County, Western Kenya
}

\author{
Joshua A. Mutanyi ${ }^{*}$ (C), Daniel O. Onguru², Sidney O. Ogolla³ and Lawrence B. Adipo²
}

\begin{abstract}
Background: Annually, 125.2 million pregnant women worldwide risk contracting malaria, including 30.3 million and 1.5 million in Sub-Saharan Africa and Kenya respectively. At least three doses of sulphadoxine pyrimethamine for intermittent preventive treatment of malaria in pregnancy (IPTp-SP) is recommended for optimal benefit. Kenya recorded low IPTp-SP optimal uptake in 2015. This study investigated the prevalence of and factors influencing IPTpSP optimal uptake in Sabatia Sub County, Western Kenya.
\end{abstract}

Methods: A cross-sectional study was conducted in Sabatia Sub County from April to October 2020. Using a validated semi-structured questionnaire, data were obtained from 372 randomly sampled post-delivery women aged 15-49 years with live birth within one year preceding the study. Women on cotrimoxazole prophylaxis during pregnancy were excluded. Pearson Chi-square and Fisher's Exact test were measures of association used. Binary logistic regression analysed predictors of optimal IPTp-SP uptake.

Results: Optimal IPTp-SP uptake was 79.6\%, 95\% Cl 75.5\%-83.7\%. Predictors of IPTp-SP optimization were gestational age at first antenatal care (ANC) visit $(P=0.04)$, frequency of ANC visits $(P<0.001)$, maternal knowledge of IPTp-SP benefits $(P<0.001)$, maternal knowledge of optimal sulphadoxine pyrimethamine $(\mathrm{SP})$ dose $(P=0.03)$ and SP administration at ANC clinic $(P=0.03)$. Late ANC initiators were less likely to receive optimal IPTp-SP $(a O R=0.4,95 \% \mathrm{Cl}$ 0.2-0.9). Odds of optimizing IPTp-SP increased among women with $\geq 4$ ANC visits (aOR $=16.7,95 \% C l$ 7.9-35.3), good knowledge of IPTp-SP benefits ( $\mathrm{aOR}=2.4,95 \% \mathrm{Cl} 1.3-4.5)$ and good knowledge of optimal SP dose $(\mathrm{aOR}=1.9,95 \%$ Cl 1.1-3.4). Women who never missed being administered SP were highly likely to receive optimal IPTp-SP $(\mathrm{aO} R=2.9$, $95 \%$ Cl 1.1-7.2)

Conclusions: This study has found high IPTp-SP optimal uptake in the study area. Efforts should be directed towards early and more frequent ANC visits. Intensive and targeted health education is required. It's fundamental to adequately stock and consistently administer SP. Future studies considering larger samples and health workers' perspectives of the health system delivery factors are recommended.

Keywords: Malaria, Pregnancy, Intermittent preventive treatment, Sulphadoxine pyrimethamine, Sabatia

*Correspondence: jmutanyi@gmail.com

${ }^{1}$ Department of Public and Community Health, School of Health

Sciences, Jaramogi Oginga Odinga University of Science and Technology, P.O. Box 210, Bondo 40601, Kenya

Full list of author information is available at the end of the article

\section{Background}

Malaria in pregnancy (MiP) remains a major public health problem. It is of considerable importance particularly when preventive interventions are inadequate [1]. 
In malaria endemic areas, MiP risk is $50 \%$ higher among pregnant women [2] due to pregnancy induced lowered immunity [3]. MiP causes anaemia, which in turn raises maternal and neonatal mortality risk [2]. Other negative consequences of MiP include haemorrhage, intrauterine growth retardation, still birth, preterm delivery, placental malaria, miscarriage, low birth weight (LBW) and congenital infections [2, 4].

Globally, 125.2 million pregnant women risk contracting malaria yearly, including 30.3 million in stable malaria transmission zones of Sub-Saharan Africa (SSA) [5]. Approximately $40 \%$ of all pregnancies in SSA would experience Plasmodium falciparum placental infection in the absence of MiP preventive interventions causing 900000 LBW deliveries annually [6]. In Africa, about 10 000 women and 75000 to 200000 infants die yearly due to $\mathrm{MiP}$ while $\mathrm{LBW}$ from $P$. falciparum infections during pregnancy causes nearly 100000 neonatal deaths [2]. In Kenya, about 1.5 million pregnancies occur yearly, with $44 \%$ in moderate to intense malaria endemic areas [3]. The country's MiP prevalence was 4918 cases per 100 000 persons in 2018 [7]. Intermittent preventive treatment of malaria in pregnancy (IPTp) with sulphadoxine pyrimethamine (SP) is recommended by World Health Organization (WHO) for controlling MiP [8].

The WHO recommends provision of IPTp-SP to all pregnant women in moderate to high malaria transmission areas starting earliest in the second trimester at each scheduled antenatal care (ANC) visit until delivery, observing at least one month interval between the SP doses [8]. Kenya's Ministry of Health recommends at least three IPTp-SP doses (IPTp-SP3+) to pregnant women in malaria endemic areas [9]. The drug is safe and can be taken with or without food. However, WHO does not recommend SP to Human Immunodeficiency Virus (HIV) infected pregnant women on cotrimoxazole prophylaxis due to the drugs' redundant mechanisms of action and synergistic worsening of adverse drug reaction [8]. SP remains cost effective and efficacious for IPTp [10, 11]. IPTp-SP has been linked to reduced MiP cases, lower placental malaria risk and reduced neonatal deaths [12]. The intervention was associated with increases in maternal haemoglobin and birth weight even in areas that experienced SP resistance in SSA [13].

Optimal IPTp-SP uptake remains below global targets in SSA [14]. Two different studies reported IPTpSP3+ uptake levels of $22 \%$ and $29.5 \%$ in Africa $[15,16]$. In Malawi and Uganda, the IPTp-SP optimal uptakes were $29.8 \%$ and $18 \%$ respectively $[17,18]$. Other studies in Ghana and Sierra Leone reported high optimal IPTpSP uptake levels of $71 \%, 76.4 \%$ and $93.24 \%$ [16, 19, 20]. In Kenya, the 2015 national survey found IPTp-SP3+ uptake to be $37.5 \%$ [21], far below the $80 \%$ national target [9].
However, IPTp-SP3+ uptake in specific areas in the country remained unknown and studies on the same after malaria policy revision in 2014 have been limited.

Socio demographic and obstetric characteristics have been found to influence the uptake of IPTp-SP by pregnant women [16-18, 22]. Knowledge related factors such as maternal knowledge of $\mathrm{MiP}$ and IPTp-SP have also been demonstrated to predict IPTp-SP optimal uptake [23, 24]. In SSA, poor IPTp-SP coverage levels and many missed opportunities for IPTp-SP delivery have been attributed to health care system inadequacies [25-27]. Despite this, determinants of IPTp-SP3+ uptake in Sabatia Sub County were unclear and unknown. This study sought to investigate the prevalence of and factors influencing IPTp-SP3+ uptake in the Sub County. Understanding the prevalence and determinants of IPTpSP3+ uptake is core for making decisions and setting priorities towards improved access to and optimization of the intervention.

\section{Methods \\ Study site and study design}

The study site was Sabatia Sub County, Vihiga County in Western Kenya. It is a malaria endemic zone. In 2018, its estimated pregnant women's population was 5117 [7]. It's mainly rural and most residents are in agriculture and rural development sector. A household-based cross-sectional study using a quantitative approach was conducted from April to October 2020. Cross-sectional study design was used due to its relevance in estimating prevalence and measuring both outcome and exposure variables simultaneously.

\section{Target and study population}

The target population was pregnant women aged 15-49 years. However, the study population included post-delivery women aged 15-49 years with live birth within one year preceding the study, were residents of Sabatia Sub County during their last pregnancy for $\geq 9$ months before the study and consented to participate. The study measured IPTp-SP uptake at pregnancy end, hence the use of post-delivery women. HIV-infected women on cotrimoxazole prophylaxis during their last pregnancy and those with mental disorders were excluded.

\section{Study variables}

The dependent variable was number of IPTp-SP doses received. Though a numerical variable, a dichotomy was created to have sub-optimal IPTp-SP uptake ( $\leq 2$ doses) 
Table 1 Definition of independent variables

\begin{tabular}{|c|c|}
\hline Variable & Definition \\
\hline \multicolumn{2}{|l|}{ Sociodemographic } \\
\hline Maternal age & Age in years, further grouped 15-24, 25-34 and 35+years \\
\hline Educational level & Highest level attained, classified as none, primary, secondary and tertiary \\
\hline Marital status & Either married or unmarried \\
\hline Residence & Either rural or urban \\
\hline Employment status & Categorized as unemployed, informal employment or formal employment \\
\hline Religion & Grouped as none, Roman Catholic, Protestant/other Christian or Muslim \\
\hline \multicolumn{2}{|l|}{ Obstetric } \\
\hline Parity & Number of live births, further categorized as 1,2 and $3+$ \\
\hline Gestational age at 1 st ANC visit & Pregnancy age at 1 st ANC visit, further grouped as $\leq 16$ or $>16$ weeks \\
\hline Frequency of ANC visit & Total number of ANC visits, further classified as $<4$ and $\geq 4$ weeks \\
\hline Experience of SP side effects & Whether a woman experienced SP side effects or not \\
\hline \multicolumn{2}{|l|}{ Knowledge related } \\
\hline Knowledge of MiP dangers & $\begin{array}{l}\geq 3 \text { correct responses indicated adequate knowledge, } 2 \text { correct responses indicated moderate knowledge, } 1 \text { cor- } \\
\text { rect response indicated low knowledge, otherwise unknowledgeable }\end{array}$ \\
\hline Knowledge of MiP prevention & $\begin{array}{l}\geq 3 \text { correct responses indicated adequate knowledge, } 2 \text { correct responses indicated moderate knowledge, } 1 \text { cor- } \\
\text { rect response indicated low knowledge, otherwise unknowledgeable }\end{array}$ \\
\hline Knowledge of IPTp-SP benefits & Identifying malaria prevention as the benefit of IPTp-SP indicated good knowledge, otherwise poor knowledge \\
\hline Knowledge of IPTp-SP start & $\begin{array}{l}\text { Mentioning gestation age between } 13 \text { to } 16 \text { weeks as the best time to start IPTp-SP indicated good knowledge, } \\
\text { otherwise poor knowledge }\end{array}$ \\
\hline Knowledge of optimal SP dose & $\begin{array}{l}\text { Mentioning uptake of SP doses in the range of } 3 \text { to } 8 \text { during the entire pregnancy period indicated good knowl- } \\
\text { edge, otherwise poor knowledge }\end{array}$ \\
\hline \multicolumn{2}{|l|}{ Health service delivery } \\
\hline SP administration at ANC clinic & Either a woman ever missed or never missed being issued SP \\
\hline ANC clinic working hours & Whether ANC services were accessible half day or whole day \\
\hline Maternal services fee & Whether a woman ever paid for ANC services or not \\
\hline ANC clinic waiting time & Time in minutes spent queueing before being attended to at ANC clinic, further grouped as $\leq 30$ and $>30$ min \\
\hline Water provision at ANC clinic & Whether ANC clinic provided water for taking SP always, sometimes or never, as reported by a pregnant woman \\
\hline Clean water at ANC clinic & Whether water for taking SP at ANC clinic was clean always, sometimes or never, as reported by a pregnant woman \\
\hline Clean cups at ANC clinic & $\begin{array}{l}\text { Whether cups/glasses for taking SP at ANC clinic were clean always, sometimes or never, as reported by a pregnant } \\
\text { woman }\end{array}$ \\
\hline Enough cups at ANC clinic & Whether cups/glasses for taking SP at ANC clinic were enough or not, as reported by a pregnant woman \\
\hline Health worker-client relationship & $\begin{array}{l}\text { On most occasions; } 1 \text {. Whether a health worker greeted a pregnant woman before attending to her, } 2 \text {. Whether } \\
\text { enough time was spent attending to the woman, } 3 \text {. Whether the woman was counselled/ educated on IPTp-SP } \\
\text { benefits, } 4 \text {. Whether the woman was given a chance to ask questions, } 5 \text {. Whether the woman felt comfortable } \\
\text { asking questions, } 6 \text {. Whether her questions were answered satisfactorily, } 7 \text {. Whether the health worker ever } \\
\text { shouted at the woman. At least four positive responses to the first six questions and a negative response to the } \\
\text { last question indicated good relationship, otherwise poor }\end{array}$ \\
\hline
\end{tabular}

and optimal IPTp-SP uptake ( $\geq 3$ doses). Table 1 shows independent variables and their definitions.

\section{Data collection}

Face to face interviews of post-delivery women by trained research assistants were conducted using validated semi-structured questionnaires (Additional file 1). Data were collected on both the dependent and independent variables. ANC clinic attendance booklets were used to verify data on SP doses received, ANC visits made and gestational age at first $\mathrm{ANC}$ visit after asking study participants on the same. Each participant's responses and booklet information were compared to determine their accuracy. All the women studied had the clinic attendance booklets.

\section{Sample size and sampling}

Sample size was calculated using Cochran's (1977) formula for categorical data. Further, Cochran's (1977) correction formula was applied since the initial obtained sample size exceeded $5 \%$ of the population $[28,29]$. The sample size was determined based on optimal IPTp-SP prevalence of $37.5 \%$ as per the 2015 national survey [21], 
a $95 \%$ confidence level, a $5 \%$ precision and a $10 \%$ nonresponse rate. The final sample size was 372 .

Simple random sampling was applied. The process began by identifying, listing and informing all potential participants about the study. After seeking permission from relevant authorities, Community Health Volunteers' registers of pregnant women were used to develop a sampling frame of 3091. Next, a consecutive identification number from 1 to 3091 was assigned to each recruited participant. Microsoft Excel (Microsoft Corporation, Redmond, Washington, US) version 2016 "RANDBETWEEN()" function was used to randomly sample 372 participants from the sampling frame.

\section{Statistical analysis of data}

Descriptive analyses of continuous and categorical variables were done by calculating means and proportions respectively. Pearson Chi-square test and Fisher's Exact test where appropriate were used to compare differences in various predictors of interest. Binary logistic regression models were fitted to determine the relationship between IPTp-SP uptake and the predictors. Predictors with $P<0.15$ from step-wise regression were included in the multivariable logistic regression model. Crude odds ratios $(\mathrm{cOR})$ and adjusted odds ratios $(\mathrm{aOR})$ were reported and all predictors with $P<0.05$ were considered to be independently associated with IPTp-SP optimal uptake. Statistical analyses were performed using Stata version 14.0 (Stata Corp., College Station, TX).

\section{Ethical considerations}

The study was approved by Jaramogi Oginga Odinga University of Science and Technology Ethics Review Committee (Approval Number: 7/17/ERC/11/3/2021) and the National Commission for Science, Technology and Innovation in Kenya (License Number: NACOSTI/P/20/5052). Informed consent was sought from all respondents using an approved consent form. Privacy and confidentiality of the study participants and all raw data were strictly observed. Sabatia Sub County Medical Officer of Health permitted the study (Additional file 1).

\section{Results}

\section{Characteristics of the respondents}

A total of 372 participants were enrolled in the study. Table 2 summarizes their general characteristics.

The participants' average age was 26.3 [Standard Deviation $(S D)=5.7]$ years, with majority $(173,46.5 \%)$ aged 25-34 years. Most $(285,76.6 \%)$ participants were married, majority $(178,47.9 \%)$ attained secondary education, about two-thirds (252, 67.7\%) were unemployed, the highest $(350,94.1 \%)$ were protestants, most $(85,22.9 \%)$
Table 2 General characteristics of participants

\begin{tabular}{|c|c|}
\hline Characteristics & $n(\%)$ \\
\hline Maternal age (years), mean ( $\pm S D$ ) & $26.3 \pm 5.7$ \\
\hline \multicolumn{2}{|l|}{ Age category } \\
\hline $15-24$ years & $160(43.0)$ \\
\hline $25-34$ years & $173(46.5)$ \\
\hline$\geq 35$ years & $39(10.5)$ \\
\hline \multicolumn{2}{|l|}{ Marital status } \\
\hline Unmarried & $87(23.4)$ \\
\hline Married & $285(76.6)$ \\
\hline \multicolumn{2}{|l|}{ Woman's education level } \\
\hline Primary & $142(38.2)$ \\
\hline Secondary & $178(47.8)$ \\
\hline Tertiary & $52(14.0)$ \\
\hline \multicolumn{2}{|l|}{ Woman's employment status } \\
\hline Not employed & $252(67.7)$ \\
\hline Informal employment & $95(25.6)$ \\
\hline Formal employment & $25(6.7)$ \\
\hline \multicolumn{2}{|l|}{ Religion } \\
\hline Catholic & $20(5.4)$ \\
\hline Protestant & $350(94.1)$ \\
\hline Muslim & $2(0.5)$ \\
\hline \multicolumn{2}{|l|}{ Ward of residence } \\
\hline Busali & $85(22.9)$ \\
\hline Wodanga & $49(13.2)$ \\
\hline North Maragoli & $31(8.3)$ \\
\hline Chavakali & $64(17.2)$ \\
\hline Sabatia & $60(16.1)$ \\
\hline Lyaduywa Izava & $83(22.3)$ \\
\hline \multicolumn{2}{|l|}{ Residence } \\
\hline Rural & $287(77.2)$ \\
\hline Urban & $85(22.8)$ \\
\hline Gestation age at 1st ANC (weeks), mean (SD) & $16.3 \pm 6.0$ \\
\hline Frequency of ANC visits, median (IQR) & $5(2)$ \\
\hline \multicolumn{2}{|l|}{ Parity } \\
\hline 1 child & $142(38.2)$ \\
\hline 2 children & $82(22.0)$ \\
\hline $3+$ children & $148(39.8)$ \\
\hline \multicolumn{2}{|l|}{ Experience of SP side effects } \\
\hline No & $197(53.0)$ \\
\hline Yes & $173(46.5)$ \\
\hline Not applicable & $2(0.5)$ \\
\hline \multicolumn{2}{|l|}{ Knowledge of MiP dangers } \\
\hline Unknowledgeable & $112(30.1)$ \\
\hline Low knowledge & $136(36.5)$ \\
\hline Moderate knowledge & $94(25.3)$ \\
\hline Adequate knowledge & $30(8.1)$ \\
\hline \multicolumn{2}{|l|}{ Knowledge of MiP prevention } \\
\hline Unknowledgeable & $5(1.3)$ \\
\hline Low knowledge & $216(58.1)$ \\
\hline Moderate knowledge & $128(34.4)$ \\
\hline
\end{tabular}


Table 2 (continued)

\begin{tabular}{ll}
\hline Characteristics & $\boldsymbol{n}(\%)$ \\
\hline Adequate knowledge & $23(6.2)$ \\
Knowledge of IPTp-SP benefits & \\
Poor & $77(20.7)$ \\
Good & $295(79.3)$ \\
Knowledge of IPTp-SP start & \\
Poor & $318(85.5)$ \\
Good & $54(14.5)$ \\
Knowledge of optimal SP dose & \\
Poor & $183(49.2)$ \\
Good & $189(50.8)$
\end{tabular}

ANC: Antenatal care; IPTp-SP: Intermittent preventive treatment of malaria in pregnancy with sulphadoxine pyrimethamine; IQR: Interquartile range; $S D$ : Standard Deviation; SP: Sulphadoxine pyrimethamine

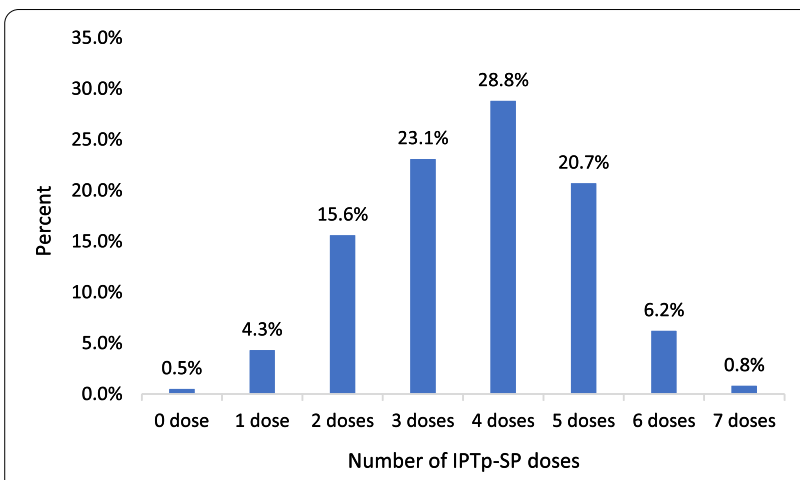

Fig. 1 Distribution of IPTp-SP uptake levels against the drug's dosage. IPTp-SP: Intermittent preventive treatment of malaria in pregnancy with sulphadoxine pyrimethamine

lived in Busali ward and over three-quarters (287, 77.2\%) were rural residents (Table 2). Mean gestation age at ANC initiation was $16.3(S D=6.0)$ weeks and median ANC visitation was 5 [Interquartile Range $(I Q R)=2$ ] visits. In total majority $(148,39.8 \%)$ had a parity of $3+$, over a half $(197,53.0 \%)$ never experienced any SP side effect, most $(136,36.6 \%)$ had low knowledge on MiP dangers, majority $(216,58.1 \%)$ had low knowledge of MiP prevention, over three-quarters $(295,79.3 \%)$ had good knowledge of IPTp-SP benefits, most (318, 85.5\%) didn't know the best IPTp-SP start date and half $(189,50.8 \%)$ had good knowledge of optimal IPTp-SP dosage (Table 2).

\section{Prevalence of IPTp-SP optimal uptake}

Overall, $(370,99.5 \%)$ of the respondents received at least one IPTp-SP dose. Of the 372 women, 79.6\%, 95\% CI 75.5\%-83.7\% received optimal IPTp-SP dose while (76, 20.4\%) took sub-optimal IPTp-SP dose. The median IPTp-SP dosage was $4(I Q R=2)$ doses. Most respondents $(107,28.8 \%)$ received four IPTp-SP doses (Fig. 1).
Socio demographic, knowledge related and obstetric factors associated with IPTp-SP uptake

This study compared characteristics of women who received optimal IPTp-SP doses to those who received sub-optimal IPTp-SP. Table 3 shows the distribution of IPTp-SP uptake against the socio-demographic, obstetric and knowledge characteristics of women during pregnancy.

Pearson Chi-square and Fisher's Exact text results show that IPTp-SP uptake was significantly associated with marital status $(P=0.04)$, gestation age at first ANC visit $(P<0.001)$, frequency of ANC visits $(P<0.001)$, knowledge of IPTp-SP benefits $(P<0.001)$ and knowledge of optimal SP dose $(P=0.01)$ (Table 3$)$.

From bivariate logistic regression analysis, marital status (cOR=1.8, 95\% CI 1.0-3.1), gestation age at first $\mathrm{ANC}$ visit (cOR=0.2, 95\% CI 0.1-0.4), frequency of ANC visits (cOR $=17.1,95 \%$ CI 9.4-31.2), knowledge of IPTpSP benefits (cOR $=2.7,95 \%$ CI 1.5-4.6) and knowledge of optimal SP dose (cOR=2.0, 95\% CI 1.2-3.3) were significant for optimal IPTp-SP uptake (Table 4). In multivariable logistic regression analysis, receipt of optimal IPTp-SP was higher among married women compared to unmarried women $(\mathrm{aOR}=1.7,95 \% \mathrm{CI} 0.9-3.1)$, but insignificant $(P=0.09)$. Gestation age at ANC initiation and frequency of ANC visits remained significant factors when others were constant $(P=0.04),(P<0.001)$ respectively. Women who started ANC attendance beyond 16 weeks gestation age were $60 \%$ less likely to receive optimal IPTp-SP (aOR=0.4, 95\% CI 0.2-0.9). Those with $\geq 4$ ANC visits were about 17 times more likely to receive optimal IPTp-SP dose (aOR $=16.7$, 95\% CI 7.935.3). Maternal knowledge of IPTp-SP benefits $(P<0.001)$ and maternal knowledge of optimal SP dose $(P=0.03)$ remained significant predictors of IPTp-SP optimization after adjusting for other covariates. Women with good knowledge of IPTp-SP benefits had over two-fold increased likelihood to receive optimal doses than those with poor knowledge $(\mathrm{aOR}=2.4,95 \%$ CI 1.3-4.5). Those with good understanding of optimal SP dose were more likely to receive IPTp-SP3 $+(\mathrm{aOR}=1.9,95 \%$ CI 1.1-3.4) (Table 4).

\section{Health service delivery factors influencing the uptake of optimal IPTp-SP}

This study compared the health service characteristics with the outcome of IPTp-SP uptake.

From Table 5, higher proportion of women who received optimal IPTp-SP never missed being administered with SP during ANC visits compared to those who received sub-optimal SP doses $(95.6 \%$ vs $86.8 \%, P=0.01)$. There was no significant association between IPTpSP uptake and water provision $(P=0.07)$, clean water 
Table 3 Socio demographic, obstetric and knowledge characteristics associated with IPTp-SP uptake

\begin{tabular}{|c|c|c|c|c|}
\hline \multirow[t]{2}{*}{ Independent variables } & \multirow{2}{*}{$\begin{array}{l}\text { All } N=372 n(\%) \\
\text { Mean } \pm S D\end{array}$} & \multicolumn{2}{|l|}{ Uptake of IPTp-SP } & \multirow[t]{2}{*}{$P$-value } \\
\hline & & $\begin{array}{l}\text { Optimal } N=296 n(\%) \\
\text { Mean } \pm S D\end{array}$ & $\begin{array}{l}\text { Sub-optimal } N=76 n(\%) \\
\text { Mean } \pm S D\end{array}$ & \\
\hline Maternal age (years), mean $( \pm S D)$ & $26.3 \pm 5.7$ & $27.5 \pm 5.8$ & $25.3 \pm 5.4$ & 0.1 \\
\hline \multicolumn{5}{|l|}{ Age category } \\
\hline 15-24 years & $160(43.0)$ & $121(40.9)$ & $39(51.3)$ & \multirow[t]{3}{*}{0.29} \\
\hline $25-34$ years & $173(46.5)$ & $142(48.0)$ & $31(40.8)$ & \\
\hline$\geq 35$ years & $39(10.5)$ & $33(11.1)$ & $6(7.9)$ & \\
\hline \multicolumn{5}{|l|}{ Marital status } \\
\hline Unmarried & $87(23.4)$ & $62(20.9)$ & $25(32.9)$ & \multirow[t]{2}{*}{$0.04^{\mathrm{a}}$} \\
\hline Married & $285(76.6)$ & $234(79.1)$ & $51(67.1)$ & \\
\hline \multicolumn{5}{|l|}{ Woman's Education level } \\
\hline Primary & $142(38.2)$ & $108(36.5)$ & $34(44.8)$ & \multirow[t]{3}{*}{0.48} \\
\hline Secondary & $178(47.8)$ & $145(49.0)$ & $33(43.4)$ & \\
\hline Tertiary & $52(14.0)$ & $43(14.5)$ & $9(11.8)$ & \\
\hline \multicolumn{5}{|l|}{ Woman's Employment status } \\
\hline Not employed & $252(67.7)$ & $193(65.2)$ & $59(77.6)$ & \multirow[t]{3}{*}{0.17} \\
\hline Informal employment & $95(25.6)$ & $82(27.7)$ & $13(17.1)$ & \\
\hline Formal employment & $25(6.7)$ & $21(7.1)$ & $4(5.3)$ & \\
\hline \multicolumn{5}{|l|}{ Religion } \\
\hline Catholic & $20(5.4)$ & $13(4.4)$ & $7(9.2)$ & \multirow[t]{3}{*}{0.15} \\
\hline Protestant & $350(94.1)$ & $282(95.3)$ & $68(89.5)$ & \\
\hline Muslim & $2(0.5)$ & $1(0.3)$ & $1(1.3)$ & \\
\hline \multicolumn{5}{|l|}{ Ward of Residence } \\
\hline Busali & 85 (22.9) & $68(23.0)$ & $17(22.4)$ & \multirow[t]{6}{*}{0.84} \\
\hline Wodanga & $49(13.2)$ & $37(12.5)$ & $12(15.8)$ & \\
\hline North Maragoli & $31(8.3)$ & $24(8.1)$ & $7(9.2)$ & \\
\hline Chavakali & $64(17.2)$ & $52(17.6)$ & $12(15.8)$ & \\
\hline Sabatia West & $60(16.1)$ & $46(15.5)$ & $14(18.4)$ & \\
\hline Lyaduywa Izava & $83(22.3)$ & $69(23.3)$ & $14(18.4)$ & \\
\hline \multicolumn{5}{|l|}{ Residence } \\
\hline Rural & $287(77.2)$ & $224(75.7)$ & $63(82.9)$ & \multirow[t]{2}{*}{0.16} \\
\hline Urban & $85(22.8)$ & $72(24.3)$ & $13(17.1)$ & \\
\hline \multicolumn{5}{|l|}{ Gestation age at 1st ANC visit } \\
\hline$\leq 16$ weeks & $197(53.0)$ & $179(60.5)$ & $18(23.7)$ & \multirow[t]{2}{*}{$<0.001^{\mathrm{a}}$} \\
\hline$>16$ weeks & $175(47.0)$ & $117(39.5)$ & $58(76.3)$ & \\
\hline \multicolumn{5}{|l|}{ Frequency of ANC visits } \\
\hline$<4$ visits & $84(22.6)$ & $33(11.2)$ & $51(67.1)$ & \multirow[t]{2}{*}{$<0.001^{\mathrm{a}}$} \\
\hline$\geq 4$ visits & $288(77.4)$ & $263(88.8)$ & $25(32.9)$ & \\
\hline \multicolumn{5}{|l|}{ Parity } \\
\hline 1 child & $142(38.2)$ & $108(36.5)$ & $34(44.7)$ & \multirow[t]{3}{*}{0.41} \\
\hline 2 children & $82(22.0)$ & $66(22.3)$ & $16(21.1)$ & \\
\hline $3+$ children & $148(39.8)$ & $122(41.2)$ & $26(34.2)$ & \\
\hline Experience of SP side effects & & & & \\
\hline No & $197(53.0)$ & $154(52.0)$ & $43(56.6)$ & 0.29 \\
\hline Yes & $173(46.5)$ & $142(48.0)$ & $31(40.8)$ & \\
\hline Not applicable & $2(0.5)$ & 0 & $2(2.6)$ & \\
\hline
\end{tabular}


Table 3 (continued)

\begin{tabular}{|c|c|c|c|c|}
\hline \multirow[t]{2}{*}{ Independent variables } & \multirow{2}{*}{$\begin{array}{l}\text { All } N=372 n(\%) \\
\text { Mean } \pm S D\end{array}$} & \multicolumn{2}{|l|}{ Uptake of IPTp-SP } & \multirow[t]{2}{*}{$P$-value } \\
\hline & & $\begin{array}{l}\text { Optimal } N=296 n(\%) \\
\text { Mean } \pm S D\end{array}$ & $\begin{array}{l}\text { Sub-optimal } N=76 n(\%) \\
\text { Mean } \pm S D\end{array}$ & \\
\hline \multicolumn{5}{|l|}{ Knowledge of MiP dangers } \\
\hline Unknowledgeable & $112(30.1)$ & $89(30.1)$ & $23(30.3)$ & \multirow[t]{4}{*}{0.97} \\
\hline Low knowledge & $136(36.5)$ & $110(37.2)$ & $26(34.2)$ & \\
\hline Moderate knowledge & $94(25.3)$ & $73(24.6)$ & $21(27.6)$ & \\
\hline Adequate knowledge & $30(8.1)$ & $24(8.1)$ & $6(7.9)$ & \\
\hline \multicolumn{5}{|c|}{ Knowledge of MiP prevention } \\
\hline Unknowledgeable & $5(1.3)$ & $4(1.4)$ & $1(1.3)$ & \multirow[t]{4}{*}{0.86} \\
\hline Low knowledge & $216(58.1)$ & $168(56.7)$ & $48(63.2)$ & \\
\hline Moderate knowledge & $128(34.4)$ & $104(35.1)$ & $24(31.6)$ & \\
\hline Adequate knowledge & $23(6.2)$ & $20(6.8)$ & $3(3.9)$ & \\
\hline \multicolumn{5}{|c|}{ Knowledge of IPTp-SP benefits } \\
\hline Poor & $77(20.7)$ & $50(16.9)$ & $27(35.5)$ & \multirow[t]{2}{*}{$<0.001^{\mathrm{a}}$} \\
\hline Good & $295(79.3)$ & $246(83.1)$ & $49(64.5)$ & \\
\hline \multicolumn{5}{|l|}{ Knowledge of IPTp-SP start } \\
\hline Poor & $318(85.5)$ & $252(85.1)$ & $66(86.8)$ & \multirow[t]{2}{*}{0.67} \\
\hline Good & $54(14.5)$ & $44(14.9)$ & $10(13.2)$ & \\
\hline \multicolumn{5}{|c|}{ Knowledge of optimal SP dose } \\
\hline Poor & $183(49.2)$ & $135(45.6)$ & $48(63.2)$ & \multirow[t]{2}{*}{$0.01^{a}$} \\
\hline Good & $189(50.8)$ & $161(53.4)$ & $28(36.8)$ & \\
\hline
\end{tabular}

${ }^{a}$ Statistically significant result at $5 \%$ significance level. ANC: Antenatal care; IPTp-SP: Intermittent preventive treatment of malaria in pregnancy with sulphadoxine pyrimethamine; SD: Standard Deviation; SP: Sulphadoxine pyrimethamine

( $P=0.38)$, clean cups $(P=0.40)$, enough cups $(P=0.46)$, ANC clinic working hours $(P=0.23)$, maternal fee $(P=0.08)$, waiting time $(P=0.34)$ and women's relationship with ANC health workers $(P=0.41)$ (Table 5).

Bivariate analysis of health service predictors of optimal IPTp-SP uptake demonstrated that women who were always provided with water (cOR $=3.4,95 \% C I 1.1-10.4)$, always provided with clean water $(\mathrm{cOR}=2.2,95 \% \mathrm{CI}$ $0.8-6.0$ ), sometimes found clean cups (cOR=3.3, 95\% $C I$ 0.3-33.9), always found clean cups (cOR=2.2, 95\% $C I$ 0.8-6.0), sometimes found enough cups (cOR $=1.2$, $95 \%$ CI 0.2-6.6), always administered with SP (cOR $=3.2$, 95\% CI 1.4-7.7), queued for $>30 \min$ (cOR=1.3, 95\% CI 0.8-2.2) and enjoyed good relationship with ANC health workers ( $\mathrm{cOR}=1.3,95 \% \mathrm{CI} 0.8-6.0)$ had increased odds of receiving optimal IPTp-SP (Table 6). However, at multivariable level, only women who never missed being issued with $\mathrm{SP}(\mathrm{aOR}=2.9,95 \% C I 1.1-7.2)$ remained significantly associated with optimal IPTp-SP uptake.

\section{Discussion}

\section{Optimal uptake of IPTp-SP}

IPTp-SP optimal uptake in the study area approaches the national target of $80 \%$ though still far from universality. This high optimal IPTp-SP uptake is consistent with studies in Ghana and Sierra Leone [19, 20, 23]. However, studies in Uganda, Tanzania and Malawi that investigated IPTp-SP optimization found low uptake levels [17, 18, 30]. The high IPTp-SP uptake prevalence could be attributable to sustained efforts by the Kenyan government and development partners towards eliminating MiP and continued investment in maternal health. For instance, maternal health care services including IPTp-SP are free. There is continuous capacity building of health workers on MiP management and prevention. Also, MiP prevention messaging is being implemented. Optimal IPTp-SP uptake in this study is higher compared to $37.5 \%$ reported in the 2015 national malaria survey [21]. Scale up of MiP preventive strategies could account for this. Besides, this may indicate geographical variations in the intervention uptake across the country. Different study methodologies could also explain the difference. For example, the survey sampled respondents from the entire country while this study drew its sample from one Sub County.

\section{Socio demographic, knowledge related and obstetric determinants of optimal IPTp-SP uptake}

In this study, late ANC initiators were less likely to receive optimal IPTp-SP. Similarly, in Tanzania first ANC booking before 17 weeks gestation age increased the 
Table 4 Socio-demographic, obstetric and knowledge predictors of uptake of SP during pregnancy

\begin{tabular}{|c|c|c|c|c|c|}
\hline \multirow[t]{3}{*}{ Predictors } & \multicolumn{2}{|l|}{ Uptake of IPTp-SP } & \multirow[t]{3}{*}{$\mathrm{cOR}, 95 \% \mathrm{Cl}$} & \multirow[t]{3}{*}{$\mathrm{aOR}, 95 \% \mathrm{Cl}$} & \multirow[t]{3}{*}{$P$-value } \\
\hline & Optimal $N=296$ & Sub-optimal $N=76$ & & & \\
\hline & $n(\%)$ & $n(\%)$ & & & \\
\hline \multicolumn{6}{|l|}{ Age category } \\
\hline $15-24$ years & $121(40.9)$ & $39(51.3)$ & Reference & & \\
\hline $25-34$ years & $142(48.0)$ & $31(40.8)$ & $1.4,0.8-2.4$ & & \\
\hline$\geq 35$ years & $33(11.1)$ & $6(7.9)$ & $1.8,0.7-4.6$ & & \\
\hline \multicolumn{6}{|l|}{ Marital status } \\
\hline Unmarried & $62(20.9)$ & $25(32.9)$ & Reference & & \\
\hline Married & $234(79.1)$ & $51(67.1)$ & $1.8,1.0-3.1$ & $1.7,0.9-3.1$ & 0.09 \\
\hline \multicolumn{6}{|l|}{ Woman's Education level } \\
\hline Primary & $108(36.5)$ & $34(44.8)$ & Reference & & \\
\hline Secondary & $145(49.0)$ & $33(43.4)$ & $1.4,0.8-2.4$ & & \\
\hline Tertiary & $43(14.5)$ & $9(11.8)$ & $1.3,0.6-2.9$ & & \\
\hline \multicolumn{6}{|c|}{ Woman's Employment status } \\
\hline Not employed & $193(65.2)$ & $59(77.6)$ & Reference & & \\
\hline Informal employment & $82(27.7)$ & $13(17.1)$ & $1.8,0.9-3.4$ & $1.7,0.9-3.3$ & 0.85 \\
\hline Formal employment & $21(7.1)$ & $4(5.3)$ & $1.6,0.5-4.9$ & $1.4,0.5-4.6$ & 0.45 \\
\hline \multicolumn{6}{|l|}{ Religion } \\
\hline Catholic & $13(4.4)$ & $7(9.2)$ & Reference & & \\
\hline Protestant & $282(95.3)$ & $68(89.5)$ & $2.2,0.8-5.7$ & $2.5,0.9-7.0$ & 0.08 \\
\hline Muslim & $1(0.3)$ & $1(1.3)$ & $0.5,0.1-10.0$ & $1.3,0.1-25.3$ & 0.87 \\
\hline \multicolumn{6}{|l|}{ Ward of Residence } \\
\hline Busali & $68(23.0)$ & $17(22.4)$ & Reference & & \\
\hline Wodanga & $37(12.5)$ & $12(15.8)$ & $0.8,0.3-1.8$ & & \\
\hline North Maragoli & $24(8.1)$ & $7(9.2)$ & $0.9,0.3-2.3$ & & \\
\hline Chavakali & $52(17.6)$ & $12(15.8)$ & $1.1,0.5-2.5$ & & \\
\hline Sabatia West & $46(15.5)$ & $14(18.4)$ & $0.8,0.3-1.7$ & & \\
\hline Lyaduywa Izava & $69(23.3)$ & $14(18.4)$ & $1.2,0.6-2.7$ & & \\
\hline \multicolumn{6}{|l|}{ Residence } \\
\hline Rural & $224(75.7)$ & $63(82.9)$ & Reference & & \\
\hline Urban & $72(24.3)$ & $13(17.1)$ & $1.6,0.8-3.1$ & $1.60,8-3.1$ & 0.62 \\
\hline \multicolumn{6}{|c|}{ Gestation age at 1st ANC visit } \\
\hline$\leq 16$ weeks & $179(60.5)$ & $18(23.7)$ & Reference & & \\
\hline$>16$ weeks & $117(39.5)$ & $58(76.3)$ & $0.2,0.1-0.4$ & $0.4,0.2-0.9$ & $0.04^{\mathrm{a}}$ \\
\hline \multicolumn{6}{|l|}{ Frequency of ANC visits } \\
\hline$<4$ visits & $33(11.2)$ & $51(67.1)$ & Reference & & \\
\hline$\geq 4$ visits & $263(88.8)$ & $25(32.9)$ & $17.1,9.4-31.2$ & $16.7,7.9-35.3$ & $<0.001^{\mathrm{a}}$ \\
\hline \multicolumn{6}{|l|}{ Parity } \\
\hline 1 child & $108(36.5)$ & $34(44.7)$ & Reference & & \\
\hline 2 children & $66(22.3)$ & $16(21.1)$ & $1.2,0.6-2.3$ & & \\
\hline $3+$ children & $122(41.2)$ & $26(34.2)$ & $1.5,0.8-2.6$ & & \\
\hline \multicolumn{6}{|c|}{ Experience of SP side effects } \\
\hline No & $154(52.0)$ & $43(56.6)$ & Reference & & \\
\hline Yes & $142(48.0)$ & $31(40.8)$ & $1.3,0.8-2.2$ & & \\
\hline Not applicable & 0 & $2(2.6)$ & $\mathrm{N} / \mathrm{A}$ & & \\
\hline \multicolumn{6}{|c|}{ Knowledge of MiP dangers } \\
\hline Unknowledgeable & $89(30.1)$ & $23(30.3)$ & Reference & & \\
\hline Low knowledge & $110(37.2)$ & $26(34.2)$ & $1.0,0.6-1.9$ & $0.7,0.4-1.5$ & 0.38 \\
\hline Moderate knowledge & 73 (24.6) & $21(27.6)$ & $0.9,0.5-1.8$ & $0.6,0.3-1.2$ & 0.13 \\
\hline
\end{tabular}


Table 4 (continued)

\begin{tabular}{|c|c|c|c|c|c|}
\hline \multirow[t]{3}{*}{ Predictors } & \multicolumn{2}{|l|}{ Uptake of IPTp-SP } & \multirow[t]{3}{*}{$\mathrm{COR}, 95 \% \mathrm{Cl}$} & \multirow[t]{3}{*}{$\mathrm{aOR}, 95 \% \mathrm{Cl}$} & \multirow[t]{3}{*}{$P$-value } \\
\hline & Optimal $N=296$ & Sub-optimal $N=76$ & & & \\
\hline & $n(\%)$ & $n(\%)$ & & & \\
\hline Adequate knowledge & $24(8.1)$ & $6(7.9)$ & $1.0,0.4-2.8$ & $0.5,0.2-1.6$ & 0.28 \\
\hline \multicolumn{6}{|c|}{ Knowledge of MiP prevention } \\
\hline Unknowledgeable & $4(1.4)$ & $1(1.3)$ & Reference & & \\
\hline Low knowledge & $168(56.7)$ & $48(63.2)$ & $0.9,0.1-8.0$ & & \\
\hline Moderate knowledge & $104(35.1)$ & $24(31.6)$ & $1.1,0.1-10.1$ & & \\
\hline Adequate knowledge & $20(6.8)$ & $3(3.9)$ & $1.2,0.1-13.7$ & & \\
\hline \multicolumn{6}{|c|}{ Knowledge of IPTp-SP benefits } \\
\hline Poor & $50(16.9)$ & $27(35.5)$ & Reference & & \\
\hline Good & $246(83.1)$ & $49(64.5)$ & $2.7,1.5-4.6$ & $2.4,1.3-4.5$ & $<0.001^{\mathrm{a}}$ \\
\hline \multicolumn{6}{|l|}{ Knowledge of IPTp-SP start } \\
\hline Poor & $252(85.1)$ & $66(86.8)$ & Reference & & \\
\hline Good & $44(14.9)$ & $10(13.2)$ & $1.2,0.6-2.5$ & & \\
\hline \multicolumn{6}{|l|}{ Knowledge of optimal SP } \\
\hline Poor & $135(45.6)$ & $48(63.2)$ & Reference & & \\
\hline Good & $161(53.4)$ & $28(36.8)$ & $2.0,1.2-3.3$ & $1.9,1.1-3.4$ & $0.03^{\mathrm{a}}$ \\
\hline
\end{tabular}

${ }^{a}$ Statistically significant result at 5\% significance level. ANC: Antenatal care; Cl: Confidence interval; COR: Crude odds ratio; IPTp-SP: Intermittent preventive treatment of malaria in pregnancy with sulphadoxine pyrimethamine; MiP: Malaria in pregnancy; SP: Sulphadoxine pyrimethamine

odds of receiving IPTp-SP3+ [30]. Consistently, two studies in Zambia and Ghana reported ANC start date as a significant predictor of optimal IPT-SP uptake [24, 31]. However, a different study in Tanzania found no relationship between ANC initiation and IPTp-SP dosage [32] though it used a smaller sample size of 138. In line with other studies in Uganda, Tanzania, Malawi, Sierra Leone and Ghana, this study found that $\geq 4$ ANC visits predicts optimal IPTp-SP uptake [17-20, 23, 24, 30]. The WHO calls for integration of IPTp-SP with initiatives for promoting focused ANC services [8]. Therefore, early ANC initiators are likely to achieve adequate visits, maximizing their contacts with health workers hence increased health education and SP administration. Evidently, pregnant women should be urged to initiate ANC visits earliest in the first trimester and adhere to all scheduled visits. Apparently, adequate ANC visits do not necessarily guarantee optimal IPTp-SP uptake. One-third of women with $\geq 4$ visits in this study received sub-optimal IPTp-SP, possibly due to health service delivery deficiencies such as drug shortage.

IPTp-SP optimization could be realized when pregnant women are adequately and properly informed of the intervention. In Tanzania, Cameroon, Zambia and Ghana, maternal knowledge of IPTp-SP positively influenced the intervention's maximum uptake [30, 31, 33, 34]. Similarly, this study found that women who understood
IPTp-SP benefits and knew the recommended SP doses were more likely to receive optimal dosage. Good maternal knowledge of IPTp-SP empowers women to develop positive attitudes and perceptions towards adequate intervention uptake.

Marital status was significant before adjusting for other covariates. The findings from this study show as it does in Ghana that married women have higher odds of taking IPTp-SP3+ [35]. Though it did not consider IPTp-SP optimization, a different study in Kenya had similar findings [36]. Possibly, married women get financial and psychosocial support from their spouses towards ANC attendance. As evidenced by a study in Bungoma East district of Kenya, women who received support from their partners were more likely to receive $>1$ SP dose [37]. It could also be that unmarried women get stigmatized for getting pregnant out of matrimony hence a need for their social protection.

Though no significant association, younger women, those with low education, the unemployed, rural residents and primiparous women had higher proportions of sub-optimal IPTp-SP uptake. As the unmarried, these are likely to be the $20.4 \%$ who received sub-optimal intervention. These women may lack adequate access to information and communication channels used for IPTp-SP promotion. Thus, mapping out these vulnerable women and using trained community health promoters to visit them, offer comprehensive MiP health 
Table 5 Health service characteristics associated with uptake of IPTp-SP

\begin{tabular}{|c|c|c|c|c|}
\hline \multirow[t]{2}{*}{ Health service delivery variables } & \multirow[t]{2}{*}{ All $N=372 n(\%)$} & \multicolumn{2}{|l|}{ Uptake of IPTp-SP } & \multirow[t]{2}{*}{$P$-value } \\
\hline & & Optimal $N=296 n(\%)$ & $\begin{array}{l}\text { Sub-optimal } \\
N=76 n(\%)\end{array}$ & \\
\hline \multicolumn{5}{|l|}{ Water provision at ANC clinic } \\
\hline Never & $4(1.1)$ & $4(1.4)$ & 0 & \multirow[t]{4}{*}{0.07} \\
\hline Sometimes & $3(0.8)$ & $3(1.0)$ & 0 & \\
\hline Always & $352(94.6)$ & $282(95.2)$ & $70(92.1)$ & \\
\hline Not applicable & $13(3.5)$ & $7(2.4)$ & $6(7.9)$ & \\
\hline \multicolumn{5}{|l|}{ Clean water at ANC clinic } \\
\hline Never & $1(0.3)$ & $1(0.3)$ & 0 & \multirow[t]{4}{*}{0.38} \\
\hline Sometimes & $2(0.5)$ & $2(0.7)$ & 0 & \\
\hline Always & $352(94.6)$ & $282(95.3)$ & $70(92.1)$ & \\
\hline Not applicable & $17(4.6)$ & $11(3.7)$ & $6(7.9)$ & \\
\hline \multicolumn{5}{|l|}{ Clean cups at ANC clinic } \\
\hline Never & $2(0.5)$ & $2(0.7)$ & 0 & \multirow[t]{4}{*}{0.4} \\
\hline Sometimes & $7(1.9)$ & $6(2.0)$ & $1(1.3)$ & \\
\hline Always & $346(93.0)$ & $277(93.6)$ & $69(90.8)$ & \\
\hline Not applicable & $17(4.6)$ & $11(3.7)$ & $6(7.9)$ & \\
\hline \multicolumn{5}{|l|}{ Enough cups at ANC clinic } \\
\hline Never & $44(11.8)$ & $37(12.5)$ & $7(9.2)$ & \multirow[t]{4}{*}{0.46} \\
\hline Sometimes & $13(3.5)$ & $11(3.7)$ & $2(2.6)$ & \\
\hline Always & $298(80.1)$ & $237(80.1)$ & $61(80.3)$ & \\
\hline Not applicable & $17(4.6)$ & $11(3.7)$ & $6(7.9)$ & \\
\hline \multicolumn{5}{|l|}{ ANC clinic working hours } \\
\hline Half day & $341(91.7)$ & $274(92.6)$ & $67(88.2)$ & \multirow[t]{2}{*}{0.23} \\
\hline Full day & $31(8.3)$ & $22(7.4)$ & $9(11.8)$ & \\
\hline \multicolumn{5}{|l|}{ SP administration at ANC clinic } \\
\hline Never missed administering & $349(93.8)$ & $283(95.6)$ & $66(86.8)$ & \multirow[t]{2}{*}{$0.01^{\mathrm{a}}$} \\
\hline Ever missed administering & $23(6.2)$ & $13(4.4)$ & $10(13.2)$ & \\
\hline \multicolumn{5}{|l|}{ Maternal service fee } \\
\hline Never paid & $363(97.6)$ & $291(98.3)$ & $72(94.7)$ & \multirow[t]{2}{*}{0.08} \\
\hline Ever paid & $9(2.4)$ & $5(1.7)$ & $4(5.3)$ & \\
\hline \multicolumn{5}{|l|}{ ANC clinic waiting time } \\
\hline$\leq 30 \mathrm{~min}$ & $214(57.5)$ & $167(56.4)$ & $47(61.8)$ & \multirow[t]{2}{*}{0.34} \\
\hline$>30 \min$ & $158(42.5)$ & $129(43.6)$ & $29(38.2)$ & \\
\hline \multicolumn{5}{|l|}{ Health worker-client relationship } \\
\hline Poor & $79(21.2)$ & $60(20.3)$ & $19(25.0)$ & \multirow[t]{2}{*}{0.41} \\
\hline Good & $293(78.8)$ & $236(79.7)$ & $57(75.0)$ & \\
\hline
\end{tabular}

${ }^{a}$ Statistically significant result at $5 \%$ significance level. ANC: Antenatal care; IPTp-SP: Intermittent preventive treatment of malaria in pregnancy with sulphadoxine pyrimethamine

education and encourage adequate ANC attendance is imperative. Information, education and communication materials developed in local dialect could buttress the above strategy. It may be desirable to facilitate peer education on MiP prevention among these vulnerable women. This study reinforces that women's perceived benefits of IPTp-SP uptake outweigh their experience of the drug's side effects. Thus, they should continue being educated that use of SP during pregnancy is safe and beneficial regardless of its side effects.

\section{Health service delivery predictors of optimal IPTp-SP uptake}

Health service delivery dynamics are critical to appropriate IPTp-SP uptake [26]. Previous studies have argued that sub-optimal IPTp-SP dosage was mainly due to health system gaps [25-27]. In this study, SP administration at ANC clinic determined IPTp-SP3+ uptake. Health workers administer SP to eligible pregnant women and offer health education during ANC attendance. Ever missing to administer SP can create a sense of mistrust among 
Table 6 Health service predictors of uptake of IPTP-SP

\begin{tabular}{|c|c|c|c|c|c|}
\hline \multirow[t]{2}{*}{ Predictors } & \multicolumn{2}{|l|}{ Uptake of IPTp-SP } & \multirow[t]{2}{*}{ cOR, 95\% Cl: } & \multirow[t]{2}{*}{ aOR, 95\% Cl: } & \multirow[t]{2}{*}{$P$-value } \\
\hline & $\begin{array}{l}\text { Optimal } N=296 \\
n(\%)\end{array}$ & $\begin{array}{l}\text { Sub-optimal } N=76 \\
n(\%)\end{array}$ & & & \\
\hline \multicolumn{6}{|l|}{ Water provision at ANC clinic } \\
\hline Never & $4(1.4)$ & 0 & N/A & & \\
\hline Sometimes & $3(1.0)$ & 0 & N/A & & \\
\hline Always & $282(95.2)$ & $70(92.1)$ & $3.4,1.1-10.4$ & $2.5,0.8-8.4$ & 0.13 \\
\hline Not applicable & $7(2.4)$ & $6(7.9)$ & Reference & & \\
\hline \multicolumn{6}{|l|}{ Clean water at ANC clinic } \\
\hline Never & $1(0.3)$ & 0 & N/A & & \\
\hline Sometimes & $2(0.7)$ & 0 & N/A & & \\
\hline Always & $282(95.3)$ & $70(92.1)$ & $2.2,0.8-6.0$ & & \\
\hline Not applicable & $11(3.7)$ & $6(7.9)$ & Reference & & \\
\hline \multicolumn{6}{|l|}{ Clean cups at ANC clinic } \\
\hline Never & $2(0.7)$ & 0 & N/A & & \\
\hline Sometimes & $6(2.0)$ & $1(1.3)$ & $3.3,0.3-33.9$ & & \\
\hline Always & $277(93.6)$ & $69(90.8)$ & $2.2,0.8-6.0$ & & \\
\hline Not applicable & $11(3.7)$ & $6(7.9)$ & Reference & & \\
\hline \multicolumn{6}{|l|}{ Enough cups at ANC clinic } \\
\hline Never & $37(12.5)$ & $7(9.2)$ & Reference & & \\
\hline Sometimes & $11(3.7)$ & $2(2.6)$ & $1.2,0.2-6.6$ & & \\
\hline Always & $237(80.1)$ & $61(80.3)$ & $0.9,0.4-2.0$ & & \\
\hline Not applicable & $11(3.7)$ & $6(7.9)$ & $0.4,0.1-1.4$ & & \\
\hline \multicolumn{6}{|l|}{ ANC clinic working hours } \\
\hline Half day & $274(92.6)$ & $67(88.2)$ & Reference & & \\
\hline Whole day & $22(7.4)$ & $9(11.8)$ & $0.6,0.3-1.4$ & $0.6,0.3-1.4$ & 0.13 \\
\hline \multicolumn{6}{|l|}{ SP administration at ANC clinic } \\
\hline Never missed administering & $283(95.6)$ & $66(86.8)$ & $3.3,1.4-7.7$ & $2.9,1.1-7.2$ & $0.03^{\mathrm{a}}$ \\
\hline Ever missed administering & $13(4.4)$ & $10(13.2)$ & Reference & & \\
\hline \multicolumn{6}{|l|}{ Maternal services fee } \\
\hline Never paid & $291(98.3)$ & $72(94.7)$ & Reference & & \\
\hline Ever paid & $5(1.7)$ & $4(5.3)$ & $0.3,0.1-1.2$ & & \\
\hline \multicolumn{6}{|l|}{ ANC clinic waiting time } \\
\hline$\leq 30 \min$ & $167(56.4)$ & $47(61.8)$ & Reference & & \\
\hline$>30 \min$ & $129(43.6)$ & $29(38.2)$ & $1.3,0.8-2.2$ & & \\
\hline \multicolumn{6}{|l|}{ Health worker-client relationship } \\
\hline Poor & $60(20.3)$ & $19(25.0)$ & Reference & & \\
\hline Good & $236(79.7)$ & $57(75.0)$ & $1.3,0.7-2.3$ & & \\
\hline
\end{tabular}

${ }^{a}$ Statistically significant result at 5\% significance level. ANC: Antenatal care; Cl: Confidence interval; COR: Crude odds ratio; IPTp-SP: Intermittent preventive treatment of malaria in pregnancy with sulphadoxine pyrimethamine; NA: Not Applicable; SP: Sulphadoxine pyrimethamine

women concerning the drug's continued availability. Thus, subsequent $\mathrm{ANC}$ visits may be missed leading to suboptimal IPTp-SP uptake. Besides, late ANC initiators who qualify for only three SP doses may receive sub-optimal dosage if they miss any dose. Inconsistent SP administration could be attributed to erratic drug supply. In Ghana, SP shortage was found to be a barrier to realizing high IPTp-SP uptake [25]. Also, health workers' inadequacies to offer intensive IPTp-SP education due to high ANC clinic workload could contribute to the inconsistency.

\section{Study limitations}

This study employed a cross sectional study design whose weakness is the impossibility to infer causation. Only women with live birth were included, missing an opportunity to study those with still birth. Responses were selfreported hence the possibility of recall bias. However, this was minimized by verification of some responses using ANC clinic attendance booklets. It was also minimized by excluding respondents with live birth beyond one year before the study. Health service delivery factors were not 
studied from health workers' perspectives. Nonetheless, this study was household-based and used documented evidence to validate some variables. Household-based approach provided a chance to study participants who would have been missed had it been hospital-based due to poor care seeking behaviour among some women.

\section{Conclusions}

This study has found high optimal IPTp-SP uptake in the study area. To sustain this, regular supportive supervision on adherence to IPTp-SP policies and guidelines is necessary. Gestational age at first ANC visit, frequency of ANC visits, maternal knowledge of IPTp-SP benefits, maternal knowledge of optimal SP dose and SP drug administration at ANC determines IPTp-SP3+ uptake. It is evident that efforts should be directed towards early and more frequent ANC visits. This study demonstrates that increasing awareness and knowledge of pregnant women on MiP preventive interventions through intensive, targeted health education and other behaviour change communication strategies is paramount to IPTpSP optimization. Consequently, it is necessary to develop and implement a comprehensive MiP health education strategy including messages on early ANC attendance, adequate $\mathrm{ANC}$ visits, adherence to all $\mathrm{ANC}$ visits, MiP dangers, MiP prevention, IPTp-SP start date, IPTp-SP benefits and optimal IPTp-SP dosage. To strengthen service delivery, it is critical for health facilities to stock adequate SP drugs and health workers should consistently administer the drugs. Future studies considering larger samples and health workers' perspectives of the health system delivery factors are recommended.

\section{Abbreviations}

ANC: Antenatal care; aOR: Adjusted odds ratio; Cl: Confidence interval; cOR: Crude odds ratio; HIV: Human immunodeficiency virus; IPTp: Intermittent preventive treatment of malaria in pregnancy; IPTp-SP: Intermittent preventive treatment of malaria in pregnancy with sulphadoxine pyrimethamine; IPTpSP3+: Optimal intermittent preventive treatment of malaria in pregnancy with sulphadoxine pyrimethamine; IQR: Interquartile range; LBW: Low birth weight; MiP: Malaria in pregnancy; N/A: Not applicable; SD: Standard deviation; SP: Sulphadoxine pyrimethamine; WHO: World Health Organization.

\section{Supplementary Information}

The online version contains supplementary material available at https://doi. org/10.1186/s40249-021-00887-4.

Additional file 1. Data were collected using a semi-structured questionnaire. Data were collected on IPTp-SP uptake; women's socio demographic, obstetric and knowledge related characteristics as well health service delivery factors.

Additional file 2. Data set used for the analysis and generation of the study results.

\section{Acknowledgements}

We are grateful to all women who participated in this study. We appreciate all Community Health Volunteers who assisted during the recruitment of study participants. Many thanks go to the Research Assistants who collected data. We are thankful to Sabatia Sub County Medical Officer of Health for permitting us to conduct the study in the Sub County.

\section{Authors' contributions}

JAM conceived, designed and implemented the study. DOO and SOO were involved in the study design, guided and supervised the implementation. LBA carried out statistical analyses. JAM drafted the manuscript. All authors read and approved the final manuscript.

\section{Funding}

No funding was received for this study.

\section{Availability of data and materials}

All data generated and used for statistical analysis during this study are included in this article (Additional file 2).

\section{Declarations}

\section{Ethics approval and consent to participate}

Ethical approval was obtained from Jaramogi Oginga Odinga University of Science and Technology Ethics Review Committee (Approval Number: 7/17/ ERC/11/3/20-21). Informed consent was sought from all respondents using an approved informed consent form.

\section{Consent for publication}

Not applicable.

\section{Competing interests}

The authors declare that they have no competing interests.

\section{Author details}

${ }^{1}$ Department of Public and Community Health, School of Health Sciences, Jaramogi Oginga Odinga University of Science and Technology, P.O. Box 210, Bondo 40601, Kenya. ${ }^{2}$ Department of Public and Community Health, School of Health Sciences, Jaramogi Oginga Odinga University of Science and Technology, P.O. Box 210, Bondo 40601, Kenya. ${ }^{3}$ Kenya Medical Research Institute, Centre for Global Health Research, P.O. Box 20778, Kisumu 00202, Kenya.

Received: 31 March 2021 Accepted: 15 July 2021

Published online: 06 August 2021

\section{References}

1. Walker PG, Floyd J, Ter Kuile F, Cairns M. Estimated impact on birth weight of scaling up intermittent preventive treatment of malaria in pregnancy given sulphadoxine-pyrimethamine resistance in Africa: a mathematical model. PLoS medicine. 2017;14(2):e1002243.

2. WHO. The Contribution of Malaria Control to maternal and new-born health. Geneva: World Health Organization (Roll Back Malaria Partnership); 2014

3. NMCP. Malaria in Pregnancy: National Malaria Control Programme; 2019 (March 2019). www.nmcp.or.ke/index.php/malaria-in-pregnancy.

4. Lagerberg RE. Malaria in pregnancy: a literature review. J Midwifery Womens Health. 2008;53(3):209-15.

5. Dellicour S, Tatem AJ, Guerra CA, Snow RW, ter Kuile FO. Quantifying the number of pregnancies at risk of malaria in 2007: a demographic study. PLoS Med. 2010;7(1):e1000221.

6. Dellicour S, Hill J, Bruce J, Ouma P, Marwanga D, Otieno P, et al. Effectiveness of the delivery of interventions to prevent malaria in pregnancy in Kenya. Malar J. 2016;15:221.

7. KHIS. Kenya Health Information System 2019 (cited 2020 January). https:// www.hiskenya.org. 
8. WHO. WHO Policy Brief for the Implementation of Intermittent Preventive Treatment of Malaria in Pregnancy Using Sulphadoxine Pyrimethamine (IPTp-SP). Geneva, Switzerland: World Health Organization; 2014.

9. MOH. The Kenya Malaria Strategy 2009-2018 (Revised 2014). Nairobi, Kenya: Ministry of Health.; 2014

10. Chico RM, Dellicour S, Roman E, Mangiaterra V, Coleman J, Menendez C, et al. Global Call to Action: maximize the public health impact of intermittent preventive treatment of malaria in pregnancy in sub-Saharan Africa. Malar J. 2015:14:207.

11. Tan KR, Katalenich BL, Mace KE, Nambozi M, Taylor SM, Meshnick SR, et al. Efficacy of sulphadoxine-pyrimethamine for intermittent preventive treatment of malaria in pregnancy, Mansa. Zambia Malar J. 2014;13:227.

12. Menendez C, Bardaji A, Sigauque B, Sanz S, Aponte JJ, Mabunda S, et al. Malaria prevention with IPTp during pregnancy reduces neonatal mortality. PloS one. 2010;5(2):e9438.

13. Desai M, Gutman J, Taylor SM, Wiegand RE, Khairallah C, Kayentao K, et al. Impact of sulfadoxine-pyrimethamine resistance on effectiveness of intermittent preventive therapy for malaria in pregnancy at clearing infections and preventing low birth weight. Clin Infect Dis. 2016:62(3):323-33.

14. Agarwal K, Alonso P, Chico RM, Coleman J, Dellicour S, Hill J, et al. Global Call to Action to scale-up coverage of intermittent preventive treatment of malaria in pregnancy: seminar report. Malar J. 2015;14:206.

15. WHO. World Malaria Report. Geneva. Switzerland: World Health Organization; 2018. p. 2018.

16. Yaya S, Uthman OA, Amouzou A, Bishwajit G. Use of intermittent preventive treatment among pregnant women in sub-Saharan Africa: evidence from malaria indicator surveys. Trop Med Infect Dis. 2018;3(1):18.

17. Azizi SC, Chongwe G, Chipukuma H, Jacobs C, Zgambo J, Michelo C. Uptake of intermittent preventive treatment for malaria during pregnancy with Sulphadoxine-Pyrimethamine (IPTp-SP) among postpartum women in Zomba District, Malawi: a cross-sectional study. BMC Pregnancy Childbirth. 2018;18(1):108.

18. Okethwangu D, Opigo J, Atugonza S, Kizza CT, Nabatanzi M, Biribawa C, et al. Factors associated with uptake of optimal doses of intermittent preventive treatment for malaria among pregnant women in Uganda: analysis of data from the Uganda Demographic and Health Survey, 2016. Malar J. 2019;18(1):250.

19. Anto F, Agongo IH, Asoala V, Awini E, Oduro AR. Intermittent preventive treatment of malaria in pregnancy: assessment of the sulfadoxinepyrimethamine three-dose policy on birth outcomes in rural Northern Ghana. J Trop Med. 2019;2019:6712685.

20. Buh A, Kota K, Bishwajit G, Yaya S. Prevalence and associated factors of taking intermittent preventive treatment in pregnancy in sierra leone. Trop Med Infect Dis. 2019;4(1):32.

21. NMCP, KNBS, ICF. Kenya Malaria Indicator Survey 2015. Nairobi, Kenya and Rockville, Maryland, USA: National Malaria Control Programme, Kenya National Bureau of Statistics and ICF International; 2016.

22. Odjidja EN, Duric P. Evaluation of demand and supply predictors of uptake of intermittent preventive treatment for malaria in pregnancy in Malawi. Malaria World J. 2017:8(20).

23. Ibrahim H, Maya ET, Issah K, Apanga PA, Bachan EG, Noora CL. Factors influencing uptake of intermittent preventive treatment of malaria in pregnancy using sulphadoxine pyrimethamine in Sunyani Municipality, Ghana. Pan African Med J. 2017;28:122.

24. Owusu-Boateng I, Anto F. Intermittent preventive treatment of malaria in pregnancy: a cross-sectional survey to assess uptake of the new sulfadoxine-pyrimethamine five dose policy in Ghana. Malar J. 2017;16(1):323.

25. Doku DT, Zankawah MM, Adu-Gyamfi AB. Factors influencing dropout rate of intermittent preventive treatment of malaria during pregnancy. BMC Res Notes. 2016;9(1):460.

26. Mafuleka T, Chuemchit M. Factors influencing utilization of intermittent preventive treatment of malaria during pregnancy among mothers of under-one children in rural Lilongwe. Malawi J Health Res. 2018:32(1):62-75

27. Thiam S, Kimotho V, Gatonga P. Why are IPTp coverage targets so elusive in sub-Saharan Africa? A systematic review of health system barriers. Malar J. 2013;12:353.

28. Bartlett JE, Kotrlik JW, Higgins CC. Organization research: determining appropriate sample size in survey research. Inf Technol Learn Perform J. 2001;19(1):43-50

29. Singh AS, Masuku MB. Sampling techniques \& determination of sample size in applied statistics research: an overview. Int J Economics, Commerce Management 2014; United Kingoma II(11).

30. Mchwampaka WM, Tarimo D, Chacky F, Mohamed A, Kishimba R, Samwel A. Factors affecting uptake of $\geq 3$ doses of Sulfadoxine-Pyrimethamine for malaria prevention in pregnancy in selected health facilities, Arusha region, Tanzania. BMC Preg childbirth. 2019. https://doi.org/10.1186/ s12884-019-2592-0

31. Sikambale $\mathrm{C}$, Halwindi $\mathrm{H}$, Baboo KS. Factors influencing utilization of intermittent presumptive treatment of malaria (IPTp) services by pregnant women in sesheke district of western province Zambia. Med J Zambia. 2013:40(1):24.

32. Ayubu MB, Kidima WB. Monitoring compliance and acceptability of intermittent preventive treatment of malaria using sulfadoxine pyrimethamine after ten years of implementation in Tanzania. Malaria Res Treatment. 2017;2017:9761289.

33. Ayiisi EA. Knowledge and utilization of intermittent preventive treatment for malaria control among pregnant women attending antenatal clinics in the Sunyani West District of Ghana. Sci J Public Health. 2017:5(3):254-62

34. Diengou NH, Cumber SN, Nkfusai CN, Mbinyui MS, Viyoff VZ, Bede F, et al. Factors associated with the uptake of intermittent preventive treatment of malaria in pregnancy in the Bamenda health districts, Cameroon. Pan African Med J. 2020:35:42.

35. Oppong FB, Gyaase S, Zandoh C, Nettey OEA, Amenga-Etego S, Anane $E A$, et al. Intermittent preventive treatment of pregnant women in Kintampo area of Ghana with sulphadoxine-pyrimethamine (SP): trends spanning 2011 and 2015. BMJ Open. 2019;9(6):e027946.

36. Choonara S, Odimegwu CO, Elwange BC. Factors influencing the usage of different types of malaria prevention methods during pregnancy in Kenya. Afr Health Sci. 2015;15(2):413-9.

37. Mutulei ACN. Factors influencing the uptake of intermittent preventive treatment for malaria in pregnancy: evidence form Bungoma East District, Kenya. Am J Public Health Res. 2013;1 (5):110-23. 